\title{
HEMIFACIAL SPASM IN LATE-ONSET MULTIPLE SCLEROSIS: A CASE REPORT AND LITERATURE REVIEW
}

Authors:

E. Onesti ${ }^{1}$

V. Frasca ${ }^{2}$

M. Inghilleri ${ }^{3}$

${ }^{1}$ Department of Neurology and Psychiatry,

Sapienza University, Rome, Italy

E-mail: emanuela.onesti@uniromal.it

${ }^{2}$ Department of Neurology and Psychiatry,

Sapienza University, Rome, Italy

E-mail: vittorio.frasca@uniroma1.it

${ }^{3}$ Department of Neurology and Psychiatry,

Sapienza University, Rome, Italy

E-mail: maurizio.inghilleri@ uniroma1.it

\section{Corresponding Author:}

Prof. Maurizio Inghilleri

Department of Neurology and Psychiatry

Viale dell’Università 30 - 00185 Rome

University of Rome "Sapienza"

Phone number: +39-06 49914120

Fax number: +39-06-49914120

Email address:

maurizio.inghilleri@uniroma1.it

\begin{abstract}
Movement disorders are rare manifestations of late-onset multiple sclerosis (LOMS). The clinical case of a 62-years old man with an acute onset of unilateral, sustained, irregular, brief contracture in his left-side facial muscles is presented. Clinical, neurophysiological and neuroimaging exams allowed a diagnosis of hemifacial spasm (HFS) in MS. Specifically, needle electromyography revealed repetitive motor unit action potentials firing at high frequency discharge (100-150 Hz) in the affected facial muscles, suggesting that demyelinating lesion involved the lower facial motor neurons in the intra-axial exit zone. Blink reflex findings confirmed the efferent demyelinating injury. In literature, HFS explicitly associated with MS are described, but not as one of the first presentation symptoms of LOMS. A careful neurophysiological evaluation can modify the interpretation of an atypical clinical presentation, allowing to establish the specific anatomical location, the extent of injury, and the most appropriate treatment.
\end{abstract}

Keywords: Hemifacial spasm; Multiple Sclerosis; Late-onset multiple sclerosis; HFS; LOMS. 


\section{Introduction}

Multiple sclerosis (MS) is an autoimmune inflammatory demyelinating disease of the central nervous system with a wide range of symptoms, depending on the location of lesions, and several pathophysiological mechanisms such as inflammation, demyelination, oxidative injury, axonal damage, remyelination, and gliosis. The diagnosis of MS involves dissemination of the lesions in time and space [1]. Patients develop a heterogeneous phenotypic expression of disease even at the same stage of the illness. The most frequent disturbances are transient or persistent pyramidal, sensory, cerebellar signs, visual dysfunction, fatigue and sphincteric impairment, leading often to important neurological disability. MS occurs usually in young adults, with disease onset between 20 and 40 years [2,3]. However, in up to $6 \%$ of cases, the first symptoms occur after the age of 50 years [4-11]. In the late-onset cases, correct diagnosis is frequently delayed whilst other possible diagnosis are being considered. The clinical course of late-onset MS (LOMS) to eliminate the entire line can be not necessarily progressive [9,12-14]. Some reports describe motor symptoms as initial signs $[4,9]$, while others find no differences between LOMS and young-onset MS population $[13,15]$.

Involuntary movement disorders are rare in MS, affecting about $1.6 \%$ of patients [16]. Tremor is the most frequent movement disorder in MS [17]. Other movement disorders are paroxysmal dystonia, chorea, ballism, myoclonus, tourettism, restless leg syndrome and hemifacial spasm (HFS).

In this study, a case report of MS with HFS due to a demyelization plaque encasing the facial nerve at its intra-axial exit zone is presented. MRI findings are exhibited and the clinical features are shown.

\section{Case report}

A 62-years-old man presented an acute onset of sustained, unilateral, irregular brief contracture of the left-side facial muscles. He noticed also sub continuous twitch-like movements in the left corner of the mouth. The disturbance was spontaneous and persisted during sleep. Symptoms were frequently 
aggravated by stress, fatigue, anxiety, and voluntary facial movements. Familial history was unremarkable. Since his adolescence, he presented migraine with visual aura. When the patient was 60 years old (two years before the clinical observation), he presented a visual disorder with a scotoma in the inferior part of his visual field. He did not perform any medical exam, and the disturbance reversed spontaneously in about three weeks.

Three weeks after the beginning of the involuntary facial movement, the patient referred to the Department of Neurology and Psychiatry, at the Center of Neuromuscular Disease in Umberto I Hospital (University of Rome "Sapienza") for investigation. On admission, neurological examination showed undulating and rippling twitch-like movements of lower facial muscles, associated with brief contracture of the left-side facial muscles producing drowning upward the angle of the mouth and prominence of the naso-labial fold. Subclinical left pyramidal signs were present. Laboratory tests including routine blood tests and auto-antibody profile were normal; needle electromyography examination of the left orbicularis oris revealed continuous resting activity of irregularly firing motor unit potentials (MUPs). Typical MUPs firing repetitively at high frequency discharge $(100-150 \mathrm{~Hz})$ in the affected facial muscles detected by electromyographic exam suggested that the lesion involved the lower motor neuron (Figure 1). Blink reflex findings were consistent with an efferent demyelinating injury (see Table). Visual evoked potentials showed a delayed P1 wave conduction in the right eye; motor evoked potentials resulted normal, while somatosensory evoked potentials by left posterior tibial nerve showed poorly formed $\mathrm{P}$ by cortical responses. The patient underwent high resolution 1.5Tesla brain and spinal cord MRI study with T2-weighted, FLAIR and T1weighted images acquired in the sagittal, axial and coronal planes; contrastenhanced images were also obtained. The MRI study showed a small, nonenhancing lesion adjacent to the left-side brain stem, near to the left emerging fibers of the facial nerve at the level of pons; other small multiple supra- and infratentorial white matter brain lesions 
(involving periventricular areas, frontal and parietal right lobes) were present (Figure 2). Moreover, three focal lesions in the spinal cord (cervical and dorsal posterolateral left side level) were observed. No lesions were enhanced by gadolinium. A diagnosis of MS with HFS caused by demyelination in the emerging fibers of the facial nerve was done. Chronic treatment with phenytoin $200 \mathrm{mg}$ two times per day resulted in rapidly progressive reduction of HFS symptoms. Almost complete recovery after 6 months of clinical follow-up was observed.

\section{Discussion}

In this case report, clinical and electrophysiological features permitted a diagnosis of HFS with involvement of the lower facial muscles due to a plaque of demyelination in the emerging fibers of the facial nerve.

HFS is a movement disorder characterized by unilateral, involuntary, irregular, painless, clonic or tonic movement of muscles innervated by the ipsilateral seventh cranial nerve (orbicularis oculi, paranasal, perioral and platysma), with synchronous spasms of the equivalent side of the face [18]. The spasms usually start as "twitching" of the lower eyelid, followed by involvement of the other muscles, leading to social uneasiness and interference with vision. Two ethiopathological theories are commonly proposed. One hypothesis provides that a pulsatile compression causes focal demyelination at the junction between the central and peripheral myelin, producing ephaptic neural impulses that travel down the facial nerve to the facial muscles. A second theory states that pulsations cause antidromic stimulation of the facial nucleus, generating epileptic activity in the same facial nucleus, causing hyperexcitability and aberrant facial nerve activity. Other infrequently (less than 2\%) causes of root compression are identified, such as cerebello-pontine angle masses, intracranial tumors, lesions in temporal bone or parotid gland, and Lyme disease.

Some cases of facial nerve injury in MS patients have been described [17,18,19-22]. Several events were associated with lesions in the region of the ipsilateral facial nucleus, and with supratentorial demyelinating lesions, while others did not have brainstem lesions but 
responded to intravenous methylprednisolone $[17,19,20,21]$. A case of bilateral alternating HFS has also been described in association with MS [23]. To the best of our knowledge, in literature, HFS explicitly associated with MS are described, but not as first presentation symptoms of LOMS $[21,24,25]$. The most common clinical presentation at onset in LOMS are motor symptoms (63\%) and sensory disorders (30\%); brainstem dysfunction occurred in $20 \%$ of patients, sphincteric and cerebellar involvement in $17 \%$ and $10 \%$ respectively, optic neuritis in $13 \%$, and cognitive disturbances in $3 \%$ [8]. Most of the LOMS patients present further neurological deficits and only $13 \%$ remains monosymptomatic $[4,7,8]$. Clinically, distinguishing the feature of HFS from other causes of involuntary facial movements, such as facial tic, myokymia, and tardive dyskinesia is important [26]. A particular differential diagnosis in MS is with the facial myokymia, a rare involuntary continuous vermicular movement extending across facial muscles [20]. Slight sustained facial contraction and paresis with significant myokymia have been described in MS [22]. In these cases the electromyography recordings showed typical myokimic discharges. Moreover, in the present clinical case, the presence of highfrequency discharges (of normalappearing MUP of 100-150 Hz) during needle EMG allows to exclude myokymic discharges, although it is not possible stated that they were absent throughout the entire clinical episode. HFS could be arguably attributed to axonal damage of the facial neurons due to demyelination of the intra-axial pontine exit zone of facial fibers. In the described patient, anatomical correlation evidenced with the neuroimaging, and fast clinical response to sodium channel-blocking antiepileptic drug phenytoin, suggests that HFS is secondary to MS. While imaging studies such as MRI may not detect the demyelination nature and slight structural abnormalities in the brain when the insult is minor or diffuse, neurophysiological testing can identify more specifically the structural brain abnormalities. In this report, EMG recordings from the left hemiface at rest, showing a continuous activity of irregularly firing MUPs in the left orbicularis oculi and orbicularis oris, and the results of the blink reflex studies, suggest a lesion in the efferent facial 
fibers at level of their intra-axial pontine zone. The paroxysms are usually considered the result of a level of demyelination insufficient to give persistent deficit, but making axons hypersensitive to minor stresses [27]. Moreover, a simultaneous supranuclear dishinibition given by supra-tentorial lesions should be excluded, because of the high-frequency discharges, the increased latency, and the absence of myokymic discharges.

In conclusion, in the present clinical case, HFS was due to an injury of the efferent fibers of the facial nerve in their intra-axial pontine exit zone caused by a plaque of demyelination, such as confirmed by electromyographic recordings. MS with clinical onset after 50 years of age can also be characterized by HFS and not only by myokimic discharges as usually reported in MS. The clinical suspect should exist in the presence of high frequency discharges and the absence of vermicular movements or continuous movements rippling.

Acknowledgments: The Authors would like to thank the patient who consented to the publishing of this case report.

\section{Disclosure of conflicts of interests:}

None of the authors have potential conflicts of interest to be disclosed.

Funding Sources: None 


\section{References}

1. Polman $\mathrm{CH}$, Reingold SC, Banwell B, et al. Diagnostic criteria for multiple sclerosis: 2010 revisions to the McDonald criteria. Ann Neurol. 2011;69:292-302 [Pubmed: 21387374].

2. Poser S, Raun NE, Poser W. Age at onset, initial symptomatology and the course of multiple sclerosis. Acta Neurol Scand. 1982;66:355-62 [Pubmed: 7136498].

3. Weinshenker BG, Bass B, Rice GP, et al. The natural history of multiple sclerosis: a geographically based study. I. Clinical course and disability. Brain. 1989;112(Pt 1):133-46 [Pubmed: 2917275].

4. Noseworthy J, Paty D, Wonnacott T, et al. Multiple sclerosis after age 50 . Neurology. 1983;33(12):1537-44 [Pubmed: 6606140].

5. Marra TR. Multiple sclerosis with onset after age 60. J Am Geriatr Soc. 1984;32:16-18 [Pubmed: 6197435].

6. Hooge JP, Redekop WK. Multiple sclerosis with very late onset. Neurology. 1992;42(10):1907-10 [Pubmed: 1407571].

7. Azzimondi G, Stracciari A, Rinaldi R, et al. Multiple sclerosis with very late onset: report of six cases and review of the literature. Eur Neurol. 1994;34(6):332-36 [Pubmed: 7851455].

8. Polliack ML, Barak Y, Achiron A. Late-onset multiple sclerosis. J Am Geriatr Soc. 2001;49(2):168-71 [Pubmed: 11207871].

9. Delalande S, De Seze J, Ferriby D, et al. Late onset multiple sclerosis. Rev Neurol (Paris). 2002;158(11):1082-87 [Pubmed: 12451340].

10. Martinelli V, Rodegher M, Moiola L, et al. Late onset multiple sclerosis: clinical characteristics, prognostic factors and differential diagnosis. Neurol Sci. 2004;25(4):S350-55 [Pubmed: 15727232].

11. Kis B, Rumberg B, Berlit P. Clinical characteristics of patients with lateonset multiple sclerosis. J Neurol. 2008;255(5):697-702 [Pubmed: 18283394].

12. Fernández-Concepción O, GómezGarcia AO, Martínez-Sobrepera HJ. Late-onset multiple sclerosis with benign course. Report of a case. Rev Neurol. 2001;33(1):94 [Pubmed: 11686144].

13. Tremlett H, Devonshire V. Is lateonset multiple sclerosis associated with a worse outcome? Neurology. 
2006;67(6):954-59 [Pubmed: $17000960]$.

14. Cossburn M, Ingram G, Hirst C, et al. Age at onset as a determinant of presenting phenotype and initial relapse recovery in multiple sclerosis. Mult Scler. 2012;18(1):45-54. [Pubmed: 21865412].

15. Lyon-Caen O, Izquierdo G, Marteau $\mathrm{R}$, et al. Late onset multiple sclerosis. A clinical study of 16 pathologically proven cases. Acta Neurol Scand. 1985;72(1):56-60 [Pubmed: 4050317].

16. Poser CM, Brinar BB. Diagnostic criteria for multiple sclerosis. Clin Neurol Neurosur. 2001; 103:1-11 [Pubmed: 11311469].

17. Nociti V, Bentivoglio AR, Frisullo G, et al. Movement disorders in multiple sclerosis: causal or coincidental association? Mult Scler. 2008;14:1284-87 [Pubmed: $18768580]$.

18. Yaltho TC, Jankovic J. The many faces of hemifacial spasm: differential diagnosis of unilateral facial spasms. Mov Disord. 2011;26:1582-92 [Pubmed: 21469208].

19. Telischi FF, Grobman LR, Sheremata WA, et al. Hemifacial spasm. Occurrence in multiple sclerosis. Arch Otolaryngol Head Neck
Surg. 1991;117(5):554-56 [Pubmed: 1850603].

20. Sarrigiannis $P$, Tsakanicas $C$, Anagnostouli M, et al. Spastic paretic hemifacial contracture (SPHC) in a patient with multiple sclerosis. A clinical, EMG and neuroimaging study. Neurophysiol Clin. 2004;34(34):147-51 [Pubmed: 15501684].

21. Koutsis G, Kokotis P, Sarrigiannis P, et al. Spastic paretic hemifacial contracture in multiple sclerosis: a neglected clinical and EMG entity. Mult Scler. 2008;14(7):927-32 [Pubmed: 18573838].

22. Mehanna R, Jankovic J. Movement disorders in multiple sclerosis and other demyelinating diseases. J Neurol Sci. 2013;328(1-2):1-8 [Pubmed: 23522528].

23. Van de Biezenbos JB, Horstink MW, van de Vlasakker CJ, et al. A case of bilateral alternating hemifacial spasms. Mov Disord. 1992;7:68-70 [Pubmed: 1557068].

24. Schnorpfeil F, Braune HJ. Nuclear facial palsy in multiple sclerosis: a case report. Electromyogr Clin Neurophysiol. 1997;37(4):207-11 [Pubmed: 9208215].

25. Tacconi P, Peltz MT, Lorefice L, et al. Facial synkinesis as a first symptom of 
Medical Research Archives, Vol. 4, Issue 7, November 2016

Hemifacial spasm in late-onset multiple sclerosis: A case report and literature review

multiple sclerosis. Mult Scler.

2016;22(11):1499-1501 [Pubmed: 26245215].

26. Potulska-Chromik A, Rudzinska M, Nojszewska M, et al. Clinical and neuroimaging correlation of movement disorders in multiple sclerosis: case series and review of the literature. Folia Neuropathol. 2014;52(1):92-100 [Pubmed: 24729346].

27. Espir M, Millac P. Paroxysmal brain stem dysfunction in multiple sclerosis. Br Med J. 1976; 18(2):1503 [Pubmed: 1000269]. 
Figure 1. Typical motor unit action potentials firing repetitively at high frequency discharge in the affected facial muscles detected by electromyography. Particular of the needle EMG recording from the orbicularis oris representing the high-frequency discharges of the same motor unit. Horizontal calibration, $10 \mathrm{~ms}$; vertical calibration, $100 \mu \mathrm{V}$.

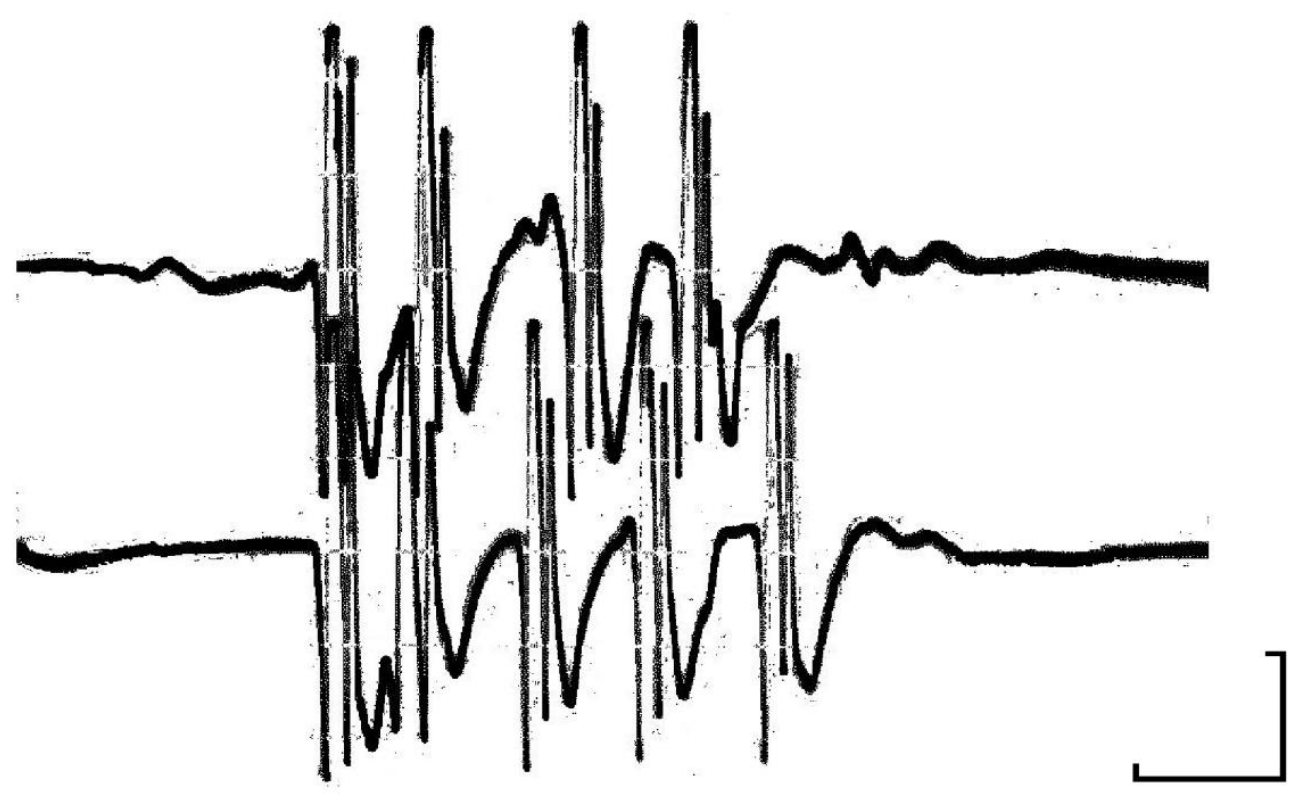


Figure 2. MRI performed with the T2-weighted sequence in the axial plain shows a nonenhancing lesion adjacent to the left-side brain stem compatible with demyelinating lesion.

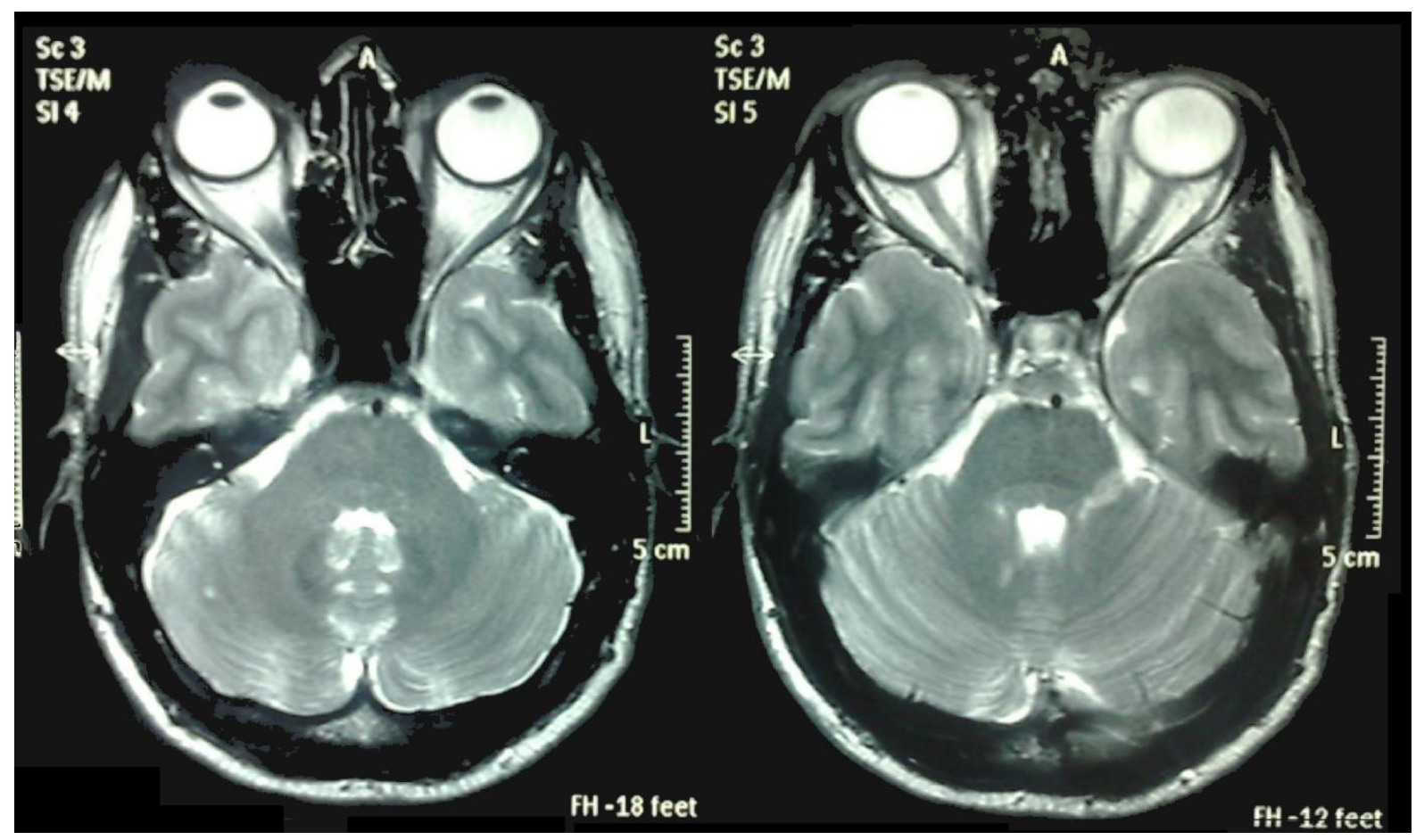

Table 1. Patient's blink reflex study. Surface recording electrodes were placed over the inferior orbicularis-oculi muscles bilaterally. Supraorbital nerve was stimulated in the superior orbital fissure with supramaximal stimulation. Latencies are expressed in millisecond (ms).

\begin{tabular}{|l|c|c|}
\hline & RIGHT STIMULATION & LEFT STIMULATION \\
\hline & & \\
\hline R1 (ms) latency & 10.2 & 13.5 \\
\hline R2 direct (ms) latency & 38.2 & 45 \\
\hline R2 crossed (ms) latency & 46.4 & 38 \\
\hline
\end{tabular}

